\title{
Rate of venous thromboembolism in a prospective all-comers cohort with COVID-19
}

\author{
Marina Rieder ${ }^{1,2}$ - Isabella Goller ${ }^{1,2} \cdot$ Maren Jeserich $^{1,2} \cdot$ Niklas Baldus $^{1,2} \cdot$ Luisa Pollmeier $^{4} \cdot$ Luisa Wirth $^{4}$. \\ Alexander Supady ${ }^{1,2} \cdot$ Christoph Bode ${ }^{1,2} \cdot$ Hans-Jörg Busch ${ }^{3} \cdot$ Bonaventura Schmid $^{3}$. Daniel Duerschmied ${ }^{1,2}$. \\ Nadine Gauchel ${ }^{1,2} \cdot$ Achim Lother ${ }^{1,2,4}$
}

Published online: 2 July 2020

(c) The Author(s) 2020

\begin{abstract}
COVID-19 is associated with a variety of clinical complications including coagulopathy, which frequently results in venous thromboembolism (VTE). Retrospective analyses reported a markedly increased rate of VTEs in COVID-19. However, most recent studies on coagulopathy in COVID-19 were only focused on critically ill patients, and without suitable control groups. We aimed to evaluate the rate of VTEs in an all-comers cohort with suspected COVID-19 during a 30-days follow-up period. We also studied the level of D-dimers and their association with the course of disease. In our prospective single-center study (DRKS00021206, 03/30/2020), we analyzed 190 patients with suspected COVID-19 admitted to the emergency department between March and April 2020. Forty-nine patients were SARS-CoV-2 positive (25.8\%). The 141 SARS-CoV-2-negative patients served as control group. After completion of a 30-days follow-up, VTE was diagnosed in 3 patients of the SARSCoV-2-positive group (6.1\%, amongst these 2 ICU cases) versus 5 patients in the SARS-CoV-2-negative group (3.5\%), however the difference was not statistically significant $(\mathrm{p}=0.427) .30$-days mortality was similar in both groups $(6.1 \%$ vs. $5 \%, \mathrm{p}=0.720$ ). Disease severity correlated with the maximum level of D-dimers during follow-up in COVID-19. The rate of VTE was numerically higher in SARS-CoV-2 positive all-comers presenting with suspected COVID-19 as compared to well-matched controls suffering from similar symptoms. VTEs in the COVID-19 group predominantly occurred in ICU courses. The maximum level of D-dimers during follow-up was associated with disease severity in COVID-19, whereas the level of D-dimers at admission was not.
\end{abstract}

Keywords COVID-19 $\cdot$ D-dimers $\cdot$ SARS-CoV-2 $\cdot$ Venous thromboembolism

\section{Highlights}

Nadine Gauchel and Achim Lother have contributed equally to this work.

Marina Rieder

marina.baeuml@universitaets-herzzentrum.de

1 Department of Medicine III (Interdisciplinary Medical Intensive Care), Medical Center, Faculty of Medicine, University of Freiburg, Freiburg, Germany

2 Department of Cardiology and Angiology I, Heart Center, University of Freiburg, Hugstetter Strasse 55, 79106 Freiburg, Germany

3 Department of Emergency Medicine, Faculty of Medicine, University Hospital of Freiburg, University of Freiburg, Freiburg, Germany

4 Institute of Experimental and Clinical Pharmacology and Toxicology, Faculty of Medicine, University of Freiburg, Freiburg, Germany
- Previous studies reported an increased incidence of VTEs in COVID-19.

- These previous studies were mostly retrospective, focused on critically ill patients and did not include suitable control groups.

- In our prospective all-comers registry, the rate of VTEs was numerically higher in COVID-19 compared to a control group of patients presenting with similar symptoms, however the difference was not statistically significant.

- The maximum level of D-dimers during follow-up was associated with disease severity in COVID-19, whereas the level of D-dimers at admission was not. 


\section{Introduction}

The novel coronavirus disease 2019 (COVID-19) first emerged in Wuhan, Province Hubei, China, in December 2019 and became pandemic since then. An infection with the diseasecausing virus SARS-CoV-2 can lead to a broad spectrum of clinical presentations, ranging from asymptomatic or mild cases to severe or even life-threatening courses with acute respiratory distress syndrome [1-3]. Since first case series from Wuhan reported an association between pulmonary embolism and COVID-19 [4], there is emerging evidence that besides pneumonia, coagulopathy is a common finding in patients suffering from COVID-19, especially in severe courses [5]. The occurrence of venous thromboembolism in serious COVID-19 infection is reported to be up to $60 \%$ [6-8] and the degree of coagulopathy was reported to be associated with disease severity [9]. However, the underlying pathomechanism causing this procoagulative state is not fully understood. It is suggested that hyperinflammation and hypoxemia lead to endothelial dysfunction and as a consequence to enhanced risk of thrombosis $[10,11]$. Interestingly, sufficient anticoagulation in severe cases reduces the risk for venous thromboembolism and is associated with a better prognosis, whereas mild or moderate courses did not seem to profit from anticoagulation [7, 12]. In general, there is a high rate of thromboembolic events despite therapeutic anticoagulation [7], however anticoagulation is strongly recommended [13].

Common laboratory markers for venous thromboembolism are D-dimers. These are cross-linked fibrin derivatives that are formed during thrombolysis. Although they are highly sensitive for venous thromboembolism, high concentrations also occur in other disorders such as infections and inflammatory environments [14]. Several studies reported elevated D-dimers in severe COVID-19 cases and their association to worse outcomes [15-19]. However, most of these studies only retrospectively analyzed COVID-19 patients undergoing ICU-therapy [20]. There is only scant data on the rate of VTE or the prognostic and diagnostic relevance of D-dimers at hospital admission and in patients on normal wards or in non-hospitalized patients.

In this study we aimed to evaluate the rate of thromboembolic events and the prognostic relevance of D-dimers in a prospective all-comers cohort of patients with suspected COVID-19. Those patients that were finally tested as positive were compared to the SARS-CoV-2 negative patients presenting with similar symptoms.

\section{Patients and methods}

We here report data from an investigator-initiated, singlecenter prospective registry study to evaluate biomarkers associated with COVID-19 (DRKS00021206, Deutsches Register klinische Studien (DRKS)) conducted at the University Medical Center-University of Freiburg.

The protocol of this study conforms to the ethical guidelines of the 1975 Declaration of Helsinki and was approved by the institutional ethical committee of the University of Freiburg (EK 153/20).

\section{Study population}

All-comers admitted to the department of emergency medicine of the University Medical Center-University of Freiburg due to suspected or proven infection with SARSCoV-2 were eligible for inclusion. The decision to perform a PCR-test for SARS-CoV-2 was made independently of study inclusion by the treating physician. Patients were asked to participate before the test results were available. Written informed consent was obtained prior to inclusion.

If patients agreed to participate, characteristics such as medical history, clinical symptoms or previous medication were recorded. Moreover, a broad spectrum of laboratory values, amongst others D-dimers, was obtained to create a biomarker profile of all study participants at the time point of hospital admission. The severity of illness was assessed in all patients using the Sequential Organ Failure Assessment (SOFA) score [21, 22].

Patients with a positive PCR-test for SARS-CoV-2 were finally allocated to the "positive" group, patients with a negative PCR-test for SARS-CoV-2 to the control group.

We performed a standardized 30-days follow-up period after study inclusion. All clinical data gathered during this period was obtained from the electronic patient file. During follow-up, no interventions were applied for the purpose of this study and all therapeutic and diagnostic procedures were applied as part of standard care at the discretion of the treating physicians. Finally, participants were contacted by phone and asked about the course of disease.

As requested from our ethics committee, we performed an interim analysis after the first 200 patients included in our registry had completed follow-up. These participants were recruited between the 26th of March 2020 and the 20th of April 2020. We had to exclude 6 patients because of inconclusive or non-available results of PCR-test. Of the 194 patients with a valid test, 42 were initially tested positive for SARS-CoV-2. Some of the patients from the SARS-CoV-2 negative group underwent further PCR-tests 
within the first days after study inclusion due to continuously suspected COVID-19. Finally, 7 of the initially negative group had a subsequent positive PCR result and had to be allocated to the positive group afterwards. In total, 49 of the 194 patients were SARS-CoV-2 positive, 145 patients were SARS-CoV-2 negative. We conducted a 30-days follow-up. 4 patients in the negative cohort were lost of follow-up as they did not want to be contacted or could not get contacted, while all positive patients completed the follow-up period. Finally, a total of 190 patients could be included in our analysis (Fig. 1).

\section{Endpoint}

The primary outcome for this analysis was the occurrence of venous thromboembolic events (VTE). VTE included pulmonary embolism (PE); deep vein thrombosis (DVT) or venous thrombosis at other sites diagnosed by accepted imaging tests. During the period considered for the present analysis, no VTE screening strategy among COVID-19 patients was in place at the study site: VTE imaging tests were only performed at the discretion of the treating physician in subjects with signs or symptoms of DVT or with an unexplained clinical worsening of e.g. the respiratory function or a rapid increase of D-dimer levels.

The length of in-hospital-/ICU-stay as well as the days on ventilation and their correlation to the maximum D-dimer levels were considered as secondary outcome measures.

\section{Data analysis}

For analysis, data were blinded to patient identity. Statistical analyses were performed using SPSS (version 25, IBM, SPSS Statistics, Armonk, USA) and GraphPad Prism 5 (GraphPad Software, San Diego, USA). Statistically significant outliers were excluded using Gubb's test. Continuous variables were tested for normal distribution by using the Shapiro-Wilk test. Data are presented as mean \pm standard deviation if found to follow a Gaussian distribution or otherwise as median with interquartile range.

Variables following Gaussian distribution were compared using student's t-test, non-normally distributed continuous values by using Mann-Whitney-U test. Categorical variables were assessed by chi-square test or Fisher's exact test as appropriate.

Correlation analysis was performed using the Spearman test for non-parametric data.

A two-tailed p-value less than 0.05 was considered statistically significant.

\section{Results}

Of the 190 patients that were finally analyzed, 49 were SARS-CoV-2 positive and 141 negative. The median age in both groups was 60 years. $61.2 \%$ of the SARS-CoV-2 positive patients were men compared to $50.4 \%$ in the SARS-CoV-2 negative group; yet the difference was not statistically significant. The SOFA-Score did not differ between both groups, indicating similar grades of illness.

We considered body mass index (BMI), smoking status, known oncological disease and previous VTE as risk factors for the development of VTEs within the study period and analyzed these parameters. There was no significant difference in any of these parameters, indicating a comparable thrombotic risk cluster in both groups. Moreover, there was no statistically significant difference in preexisting anticoagulation.

The patients differed significantly in the symptoms presented at admission: dyspnea was more common in the SARS-CoV2 negative group ( $41.7 \%$ vs. $52.4 \%, \mathrm{p}=0.002)$, whereas cough $(58.3 \%$ vs. $37.6 \%, \mathrm{p}<0.0001)$ and fever (75\% vs. $48.2 \%, \mathrm{p}<0.0001)$ could be observed more frequently in the COVID-19 patients. The rate of hospital admissions tended to be higher in the SARS-CoV-2 positive group (81.6\% vs. $66.7 \%, \mathrm{p}=0.068)$ (Table 1$)$.

A native CT-scan was performed in 4 patients $(8.2 \%)$ in the SARS-CoV-2 positive group compared to 8 patients $(5.7 \%)$ in the negative group $(\mathrm{p}=0.374)$. CT-pulmonary Angiography (CTPA) was performed in 5 patients $(10.2 \%)$ in the SARS-CoV-2 positive group compared to 14 patients $(9.9 \%)$ in the negative group $(\mathrm{p}=1.0)$. The rate of VTEs diagnosed at admission was 0 in the SARS-CoV-2 positive group vs. 3 in the SARS-CoV-2 negative group; however the difference was not statistically significant.

D-Dimers at admission did not differ between both groups $(1.1 \pm 1.4 \mathrm{mg} / \mathrm{l}$ vs. $0.8 \pm 1.7 \mathrm{mg} / \mathrm{l}, \mathrm{p}=0.2995)$ (Table 1).

After completion of the 30-days follow-up period, a total of 3 VTEs had occurred in the SARS-CoV-2 positive group (6.1\%). 2 of these 3 patients had suffered pulmonary embolism, 1 was diagnosed with a thrombosis of the portal vein. The two patients with the diagnosis of pulmonary embolism had been admitted to ICU (patient 1: 20 days in hospital, 9 days on ICU; patient 2: hospitalized during the whole follow-up period, 27 days on ICU). The patient with the thrombosis of the portal vein stayed in hospital for a total of 7 days, but was not admitted to ICU. In the SARS-CoV-2 negative group, a total of 5 patients were diagnosed with pulmonary embolism $(3.5 \%)$. All of them had been hospitalized for at least 10 days, but none was admitted to ICU. The difference in total VTE between both groups was not statistically significant $(p=0.427)$. 


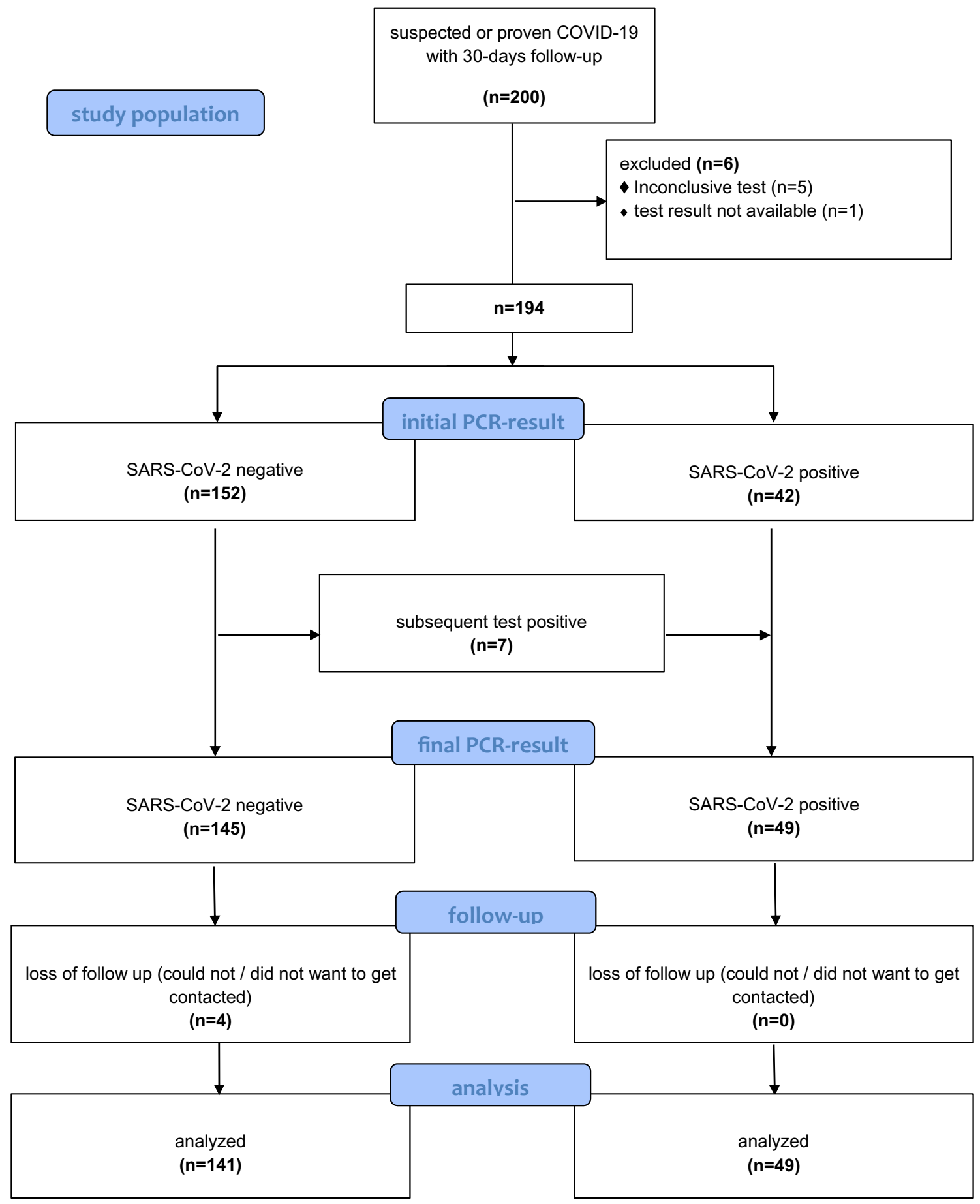

Fig. 1 Schematic representation of the allocation to the positive or negative group of the first 200 participants included in our study. Six patients had to be excluded due to inconclusive or non-available test results. Seven of the initially negative group had a subsequent positive PCR result and had to be allocated to the positive group after-

Moreover, the maximum level of D-dimers during follow-up did not differ between both groups $(1.2 \pm 3.3$ vs. $2.1 \pm 3.5, \mathrm{p}=0.8819$ ). wards. Four participants of the SARS-CoV-2 negative group were lost of follow-up, while all positive patients completed follow-up. Finally, 141 negative and 49 positive participants were included in the analysis. The flow diagram is based on the template of the CONSORT flow diagram [30-32]

We observed that SARS-CoV-2 positive patients stayed markedly longer in hospital $(10 \pm 12$ vs. $5 \pm 12, p=0.0006)$, tended to be more often admitted to ICU $(16.3 \%$ vs. $7.1 \%$, 
Table 1 Patient characteristics at hospital admission

\begin{tabular}{llll}
\hline & $\begin{array}{l}\text { SARS-CoV-2 positive } \\
(\mathrm{n}=49)\end{array}$ & $\begin{array}{l}\text { SARS-CoV-2 negative } \\
(\mathrm{n}=141)\end{array}$ & p-value \\
\hline Patients characteristics & & & \\
Age [years] & $60 \pm 23^{\mathrm{a}}$ & $60 \pm 33^{\mathrm{a}}$ & $0.6499^{\mathrm{c}}$ \\
Sex [male] & $30(61.2 \%)^{\mathrm{b}}$ & $71(50.4 \%)^{\mathrm{b}}$ & $0.245^{\mathrm{d}}$ \\
BMI [kg/m ${ }^{2}$ ] & $26.6 \pm 6.6^{\mathrm{a}}$ & $24.6 \pm 7,62^{\mathrm{a}}$ & $0.3558^{\mathrm{c}}$ \\
Smoking & $5(10.6 \%)^{\mathrm{b}}$ & $27(19.4 \%)^{\mathrm{b}}$ & $0.188^{\mathrm{d}}$ \\
Symptoms at admission & & & \\
Dyspnoe & $20(41.7 \%)^{\mathrm{b}}$ & $74(52.4 \%)^{\mathrm{b}}$ & $0.002^{\mathrm{d}}$ \\
Cough & $28(58.3 \%)^{\mathrm{b}}$ & $53(37.6 \%)^{\mathrm{b}}$ & $<0.0001^{\mathrm{d}}$ \\
Fever & $36(75 \%)^{\mathrm{b}}$ & $68(48.2 \%)^{\mathrm{b}}$ & $<0.0001^{\mathrm{d}}$ \\
SOFA-Score & $1 \pm 2.75^{\mathrm{a}}$ & $1 \pm 33^{\mathrm{a}}$ & $0.8767^{\mathrm{c}}$ \\
Medical history & & & \\
Oncological disease & $11(22.4 \%)^{\mathrm{b}}$ & $46(32.6 \%)^{\mathrm{b}}$ & $0.208^{\mathrm{d}}$ \\
Previous VTE & $6(12.2 \%)^{\mathrm{b}}$ & $15(10.8 \%)^{\mathrm{b}}$ & $0.887^{\mathrm{d}}$ \\
Preexisting anticoagulation & $6(12.2 \%)$ & $26(18.8 \%)$ & $0.222^{\mathrm{d}}$ \\
Heparine (therapeutic) & $1(2.0 \%)^{\mathrm{b}}$ & $2(1.4 \%)^{\mathrm{b}}$ & $0.594^{\mathrm{d}}$ \\
DOAK & $4(8.1 \%)^{\mathrm{b}}$ & $17(12.3 \%)^{\mathrm{b}}$ & $0.592^{\mathrm{d}}$ \\
VKA & $1(2.0 \%)^{\mathrm{b}}$ & $7(5.1 \%)^{\mathrm{b}}$ & $0.624^{\mathrm{d}}$ \\
Diagnostics/diagnosis at admission & & & \\
D-dimers [mg/l] & $1.1 \pm 1.4^{\mathrm{a}}$ & $0.8 \pm 1.7^{\mathrm{a}}$ & $0.2995^{\mathrm{c}}$ \\
Native CT-scan & $4(8.2 \%)^{\mathrm{b}}$ & $8(5.7 \%)^{\mathrm{b}}$ & $0.374^{\mathrm{d}}$ \\
CTPA & $5(10.2 \%)^{\mathrm{b}}$ & $14(9.9 \%)^{\mathrm{b}}$ & $1.0^{\mathrm{d}}$ \\
VTE diagnosed at admission & $0(0 \%)^{\mathrm{b}}$ & $3(2.1 \%)^{\mathrm{b}}$ & $0.570^{\mathrm{d}}$ \\
Hospital admission & $40(81.6 \%)^{\mathrm{b}}$ & $94(66.7 \%)^{\mathrm{b}}$ & $0.068^{\mathrm{d}}$ \\
\hline
\end{tabular}

p-values refer to the comparison between the SARS-CoV-2 negative and the SARS-CoV-2 positive patients ${ }^{\text {a }}$ Presented as median \pm interquartile range

${ }^{\mathrm{b}}$ Number of patients (with percentage based on the number of patients with a non-missing value for that characteristic)

${ }^{c}$ Based on Mann-Whitney-U test for nonparametric variables

${ }^{\mathrm{d}}$ Based on chi-square test/Fisher's exact test as appropriate for categorical variables $\mathrm{p}=0.085)$ and stayed longer on ICU (16.8 \pm 9.7 vs. $3.8 \pm 2.4$, $\mathrm{p}=0.0038)$ than participants of the control group. COVID19 patients were more often in need of invasive ventilation $(12.2 \%$ vs. $2.1 \%, \mathrm{p}=0.01)$. The 30 -days mortality however did not differ between both groups $(6.1 \%$ vs. $5 \%, \mathrm{p}=0.720)$ (Table 2).

As mentioned above, the levels of D-dimers at admission and the maximum level during follow-up did not differ between both groups. The D-dimers at admission did not correlate with disease severity measured as days at hospital, days on ICU and length of on non-invasive or invasive ventilation in the SARS-CoV-2 positive patients. However, the length of hospital stay positively correlated with the level of D-dimers at hospital admission in the SARS-CoV-2 negative group.

In contrast to the level of D-dimers at admission, the maximum levels of $\mathrm{D}$-dimers during the 30-days follow-up period correlated significantly with the severity of disease in the SARS-CoV-2 positive group whereas they did not correlate with days at hospital, days on ICU and days on non-invasive or invasive ventilation in the control group (Table 3).

\section{Discussion}

We here report data from the first 200 all-comers with suspected or proven SARS-CoV-2 infection included in our prospective single-center registry study to evaluate biomarkers associated with COVID-19. We analyzed the data in regards of VTEs and evaluated the association between D-dimers and disease severity.

Our study collective consisted of all-comers presenting at the emergency department with symptoms such as fever, cough or dyspnea that were suspicious of COVID-19. As most currently available data on COVID-19 is based on retrospective analyses, the prospective design and the comparison between SARS-CoV-2 positive patients to a control 
Table 2 Outcome and patient characteristics after 30 days follow-up
Table 3 Correlation analysis
SARS-CoV-2 positive

SARS-CoV-2

negative

\begin{tabular}{llll}
\hline Hospital stay & & & \\
$\quad$ Days at hospital & $10 \pm 12^{\mathrm{a}}$ & $5 \pm 12^{\mathrm{a}}$ & $0.0006^{\mathrm{e}}$ \\
Admitted to ICU & $8(16.3 \%)^{\mathrm{c}}$ & $10(7.1 \%)^{\mathrm{c}}$ & $0.085^{\mathrm{f}}$ \\
Days on ICU & $16.8 \pm 9.7^{\mathrm{b}}$ & $3.8 \pm 2.4^{\mathrm{b}}$ & $0.0038^{\mathrm{d}}$ \\
Non-invasive ventilation & $3(6.1 \%)^{\mathrm{c}}$ & $2(1.4 \%)$ & $0.109^{\mathrm{f}}$ \\
Invasive ventilation & $6(12.2 \%)^{\mathrm{c}}$ & $3(2.1 \%)^{\mathrm{c}}$ & $0.01^{\mathrm{f}}$ \\
VTE diagnosis/diagnostics & & & \\
Maximum level of D-dimers [mg/l] & $1.2 \pm 3.3^{\mathrm{a}}$ & $2.1 \pm 3.5^{\mathrm{a}}$ & $0.8819^{\mathrm{e}}$ \\
$\quad$ during follow-up & & & \\
Native CT-scan & $7(14.3 \%)^{\mathrm{c}}$ & $9(6.4 \%)^{\mathrm{c}}$ & $0.131^{\mathrm{f}}$ \\
CTPA & $8(16.3 \%)^{\mathrm{c}}$ & $27(19.1 \%)^{\mathrm{c}}$ & $0.831^{\mathrm{f}}$ \\
VTE total & $3(6.1 \%)^{\mathrm{c}}$ & $5(3.5 \%)^{\mathrm{c}}$ & $0.427^{\mathrm{f}}$ \\
30-days mortality & $3(6.1 \%)^{\mathrm{b}}$ & $7(5 \%)^{\mathrm{b}}$ & $0.720^{\mathrm{f}}$ \\
\hline
\end{tabular}

p-values refer to the comparison between the SARS-CoV-2 negative and the SARS-CoV-2 positive patients ${ }^{\text {a }}$ Presented as median \pm interquartile range

${ }^{\mathrm{b}}$ Presented as mean \pm standard deviation

${ }^{\mathrm{c}}$ Number of patients (with percentage based on the number of patients with a non-missing value for that characteristic)

${ }^{\mathrm{d}}$ Based on student's t-test for variables following a Gaussian distribution

${ }^{\mathrm{e}}$ Based on Mann-Whitney-U test for nonparametric variables

${ }^{\mathrm{f}}$ Based on chi-square test/Fisher's exact test as appropriate for categorical variables

\begin{tabular}{lll}
\hline & SARS-CoV-2 positive & $\begin{array}{l}\text { SARS-CoV-2 } \\
\text { negative }\end{array}$ \\
\hline $\begin{array}{l}\text { D-Dimers at admission } \\
\text { Days at hospital }\end{array}$ & $\mathrm{r}=0.201$ & $\mathbf{r}=\mathbf{0 . 6 3 1}$ \\
& $\mathrm{p}=0.287$ & $\mathbf{p}=<\mathbf{0 . 0 0 1}$ \\
Days on ICU & $\mathrm{r}=-0.32$ & $\mathrm{r}=0.131$ \\
& $\mathrm{p}=0.867$ & $\mathrm{p}=0.207$ \\
Days on non-invasive ventilation & $\mathrm{r}=0.124$ & $\mathrm{r}=0.134$ \\
& $\mathrm{p}=0.514$ & $\mathrm{p}=0.197$ \\
Days on invasive ventilation & $\mathrm{r}=-0.093$ & $\mathrm{r}=0.048$ \\
Maximum of D-dimers during follow-up & $\mathrm{p}=0.626$ & $\mathrm{p}=0.646$ \\
Days at hospital & & $\mathrm{r}=0.419$ \\
Days on ICU & $\mathbf{r}=\mathbf{0 . 3 9 8}$ & $\mathrm{p}=0.106$ \\
Days on non-invasive ventilation & $\mathbf{p}=\mathbf{0 . 0 2 9}$ & $\mathrm{r}=0.064$ \\
& $\mathbf{r}=\mathbf{0 . 5 5 0}$ & $\mathrm{p}=0.814$ \\
Days on invasive ventilation & $\mathbf{p}=\mathbf{0 . 0 0 2}$ & $\mathrm{r}=-0.140$ \\
& $\mathrm{r}=0.316$ & $\mathrm{p}=0.605$ \\
& $\mathrm{p}=0.089$ & $\mathrm{r}=-0.140$ \\
\hline
\end{tabular}

The level of D-dimers at hospital admission and the maximum level during follow-up were correlated with days at hospital, days on ICU, days on non-invasive ventilation or days on invasive ventilation. As all parameters were non-normally distributed, Spearman correlation analysis was performed for all tests. A p-value less than 0.05 was considered statistically significant (bold). 
collective presenting with similar symptoms are a strength of our study.

All patients analyzed for this manuscript were included in the study during the peak of the COVID-19 pandemic in Germany in March/April 2020. In total, $1 / 4$ of all patients presenting with the above-mentioned symptoms at our emergency department during these months suffered from COVID-19. Interestingly, more than $10 \%$ of all positive participants were initially tested negative, but received another SARS-CoV-2 test within the next days due to persistent symptoms suspicious for COVID-19 and were finally proven positive. These findings show that repeated testing is indicated if PCR result is initially negative but patients present with clinically suspected COVID- 19.

In our collective, the SARS-CoV-2 positive and negative patients did not differ in most baseline characteristics. Age, sex, severity of disease assessed by SOFA-Score and risk factors for VTEs such as BMI, smoking status, preexisting anticoagulation or oncological diseases were similar in both groups. Finally, the rate of VTEs in the COVID-19 cohort was numerically higher in the SARS-CoV-2 positive group, however the difference was not statistically significant. Previous reports described that patients suffering from COVID-19 present a high rate of venous and arterial thromboembolic events, partly even despite prophylactic or therapeutic anticoagulation [7, 8, 23]. However, all these previous studies were retrospectively analyzing critically ill patients with COVID-19 requiring ICU-therapy. None of these reports was focused on all-comers and none of them had prospectively included a control collective. Yet, consistent with these previous descriptions that critically ill patients with COVID-19 frequently develop VTEs, the 2 patients of our positive group diagnosed with pulmonary embolism had been admitted to ICU prior to VTE diagnosis. None of the VTE-patients of our control group required ICU-therapy.

D-dimers at admission and the maximum levels during follow-up did not differ between our study groups. Median D-dimers at admission were $1.1 \mathrm{mg} / \mathrm{dl}$ in our COVID-19 group. This is in line with previous studies from Wuhan reporting D-dimer levels at admission between $0.2-1.4 \mathrm{mg}$ / $\mathrm{dl}$ in SARS-CoV-2 positive patients [24, 25].

A previous study reported elevated D-dimers in COVID-19 positive patients compared to controls [26], however the authors described COVID-19 patients in contrast to healthy controls, whereas we compared SARSCoV-2 positive patients with negative participants presenting with similar symptoms. Moreover, the level of D-dimers in SARS-CoV-2 positive patients reported by the authors $(10.36 \mathrm{mg} / \mathrm{l})$ was markedly elevated compared to the D-dimers in our all-comers cohort that was comparable to other analyses from China as reported above. In our SARS-CoV-2 positive collective, D-dimers at admission did not correlate with disease severity depicted by days in hospital, days on ICU and days on ventilation. Previous retrospective studies from Wuhan claimed that elevated D-dimers at admission could effectively predict ICU-admission or in hospital-mortality [24, 25]. Yet these were retrospective trials with most probably selection bias, as not all patients received D-dimer testing at admission and only those that did for different reasons were included [15]. In contrast to this study, our trial had a prospective design with a control group and we determined D-dimers at admission independently of clinically suspected VTE.

The maximum level of D-dimers correlated significantly with the days in hospital, the length of ICU-stay and duration of ventilation in the COVID-19 cohort. This is in line with a variety of previous studies reporting that D-dimers were elevated in critically ill hospitalized SARSCoV-2 patients if compared to less severe courses [18, 19, 27]. A recent pooled analysis also reported an association of D-dimer levels to disease severity [20].

The mortality in the Covid-19 group did not differ from the SARS-CoV-2 negative participants. This is in contrast to a previous retrospective analysis comparing the mortality of patients with COVID-19 pneumonia to SARS-CoV-2 negative patients with pneumonia. This retrospective analysis from Wuhan reported a markedly elevated mortality in SARS-CoV-2 positive patients compared to the controls [28]. However, the study from Wuhan was a retrospective analysis, whereas we prospectively compared COVID-19 patients to negative participants presenting with similar symptoms.

The case-fatality rate in Germany is currently $4.7 \%$ [29]. Mortality in our SARS-CoV-2 positive study group was slightly higher $(6.1 \%), 80 \%$ of our patients were hospitalized. Compared to other parts of the world, the mortality rate in Germany is relatively lower. For example, case fatality rate in Canada is currently $8.2 \%$ and in France $15.2 \%$ [29]. A number of reasons may explain these differences, including different healthcare systems.

\section{Conclusion}

In our prospective single-center registry study, the rate of VTEs was numerically higher in SARS-CoV-2 positive all-comers presenting with suspected COVID-19 as compared to well-matched controls suffering from similar symptoms. However, the difference in our study was not statistically significant. VTEs in the SARS-CoV-2 positive group mostly occurred in the severe courses. The maximum level of D-dimers during follow-up was associated with disease severity in COVID-19, whereas the level of D-dimers at admission was not. 


\section{Limitations}

Regarding the results of our analysis, we have to consider the comparatively low number of SARS-CoV-2 positive patients (49 in total) and the low number of participants with severe course of disease. Moreover, we did not screen our collective systematically for VTEs. Only clinically apparent VTEs were considered. However, the prospective study design comparing COVID-19 patients with a control group presenting with similar symptoms is an extraordinary strength of our study. To our knowledge, nearly all previous reports on COVID-19 were based on retrospective analyses and mostly without any control groups.

Acknowledgements Open Access funding provided by Projekt DEAL.

Author contributions MR, DD, AL and NG designed the study and the interim analysis. Data entry into our database and data evaluation were conducted by MR, IG, MJ, NB, LP and LW. BS, H-JB, AS and CB supported in study design, data entry and evaluation. MR and NG wrote the manuscript.

Funding M Rieder is funded by the IMM-PACT-Programme for Clinician Scientists, Department of Medicine II, Medical Center-University of Freiburg and Faculty of Medicine, University of Freiburg, funded by the Deutsche Forschungsgemeinschaft (DFG, German Research Foundation) - 413517907. A Lother is funded by the BertaOttenstein-Programme for Advanced Clinician Scientists, Faculty of Medicine, University of Freiburg.

Availability of data and material All datasets used in this manuscript are available from the corresponding author on reasonable request.

\section{Compliance with ethical standards}

Conflict of interest All authors declared no conflicts of interests. All authors agreed to publish this manuscript.

Ethical approval The protocol of this study conforms to the ethical guidelines of the 1975 Declaration of Helsinki and was approved by the institutional ethical committee of the University of Freiburg (EK $153 / 20)$.

Consent to participate Written informed consent was obtained from all participants prior to inclusion.

Open Access This article is licensed under a Creative Commons Attribution 4.0 International License, which permits use, sharing, adaptation, distribution and reproduction in any medium or format, as long as you give appropriate credit to the original author(s) and the source, provide a link to the Creative Commons licence, and indicate if changes were made. The images or other third party material in this article are included in the article's Creative Commons licence, unless indicated otherwise in a credit line to the material. If material is not included in the article's Creative Commons licence and your intended use is not permitted by statutory regulation or exceeds the permitted use, you will need to obtain permission directly from the copyright holder. To view a copy of this licence, visit http://creativecommons.org/licenses/by/4.0/.

\section{References}

1. Wu Z, McGoogan JM (2020) Characteristics of and important lessons from the Coronavirus disease 2019 (COVID-19) outbreak in China: Summary of a Report of 72314 Cases From the Chinese Center for Disease Control and Prevention. JAMA 323:1239-1242. https://doi.org/10.1001/jama.2020.2648

2. Wang D, Hu B, Hu C et al (2020) Clinical characteristics of 138 hospitalized patients with 2019 Novel Coronavirus-infected pneumonia in Wuhan, China. JAMA 323:1061-1069. https:// doi.org/10.1001/jama.2020.1585

3. Guan W, Ni Z, Hu Y et al (2020) Clinical characteristics of Coronavirus disease 2019 in China. N Engl J Med. https://doi. org/10.1056/NEJMoa2002032

4. Xie Y, Wang X, Yang P, Zhang S (2020) COVID-19 complicated by acute pulmonary Embolism. Radiol Cardiothorac Imaging 2:e200067. https://doi.org/10.1148/ryct.2020200067

5. Pavoni V, Gianesello L, Pazzi M et al (2020) Evaluation of coagulation function by rotation thromboelastometry in critically ill patients with severe COVID-19 pneumonia. J Thromb Thrombolysis. https://doi.org/10.1007/s11239-020-02130-7

6. Wichmann D, Sperhake J-P, Lütgehetmann M et al (2020) Autopsy findings and venous thromboembolism in patients with COVID-19: a prospective cohort study. Ann Intern Med. https ://doi.org/10.7326/M20-2003

7. Llitjos J, Leclerc M, Chochois C et al (2020) High incidence of venous thromboembolic events in anticoagulated severe COVID-19 patients. J Thromb Haemost JTH. https://doi. org/10.1111/jth.14869

8. Lodigiani C, Iapichino G, Carenzo L et al (2020) Venous and arterial thromboembolic complications in COVID-19 patients admitted to an academic hospital in Milan, Italy. Thromb Res 191:9-14. https://doi.org/10.1016/j.thromres.2020.04.024

9. Fogarty H, Townsend L, Ni Cheallaigh C et al (2020) COVID19 Coagulopathy in Caucasian patients. Br J Haematol. https:// doi.org/10.1111/bjh.16749

10. Henry BM, Vikse J, Benoit S et al (2020) Hyperinflammation and derangement of renin-angiotensin-aldosterone system in COVID-19: A novel hypothesis for clinically suspected hypercoagulopathy and microvascular immunothrombosis. Clin Chim Acta Int J Clin Chem 507:167-173. https://doi.org/10.1016/j. cca.2020.04.027

11. Panigada M, Bottino N, Tagliabue P et al (2020) Hypercoagulability of COVID-19 patients in Intensive Care Unit. A report of thromboelastography findings and other parameters of hemostasis. J Thromb Haemost JTH. https://doi.org/10.1111/jth.14850

12. Tang N, Bai H, Chen X et al (2020) Anticoagulant treatment is associated with decreased mortality in severe coronavirus disease 2019 patients with coagulopathy. J Thromb Haemost JTH 18:1094-1099. https://doi.org/10.1111/jth.14817

13. Bikdeli B, Madhavan MV, Gupta A et al (2020) Pharmacological agents targeting thromboinflammation in COVID-19: review and implications for future research. Thromb Haemost. https://doi. org/10.1055/s-0040-1713152

14. Kelly J, Hunt BJ (2002) Role of D-dimers in diagnosis of venous thromboembolism. Lancet 359:456-458. https://doi.org/10.1016/ S0140-6736(02)07669-9

15. Gris J-C, Quéré I, Pérez-Martin A et al (2020) Uncertainties on the prognostic value of D-dimers in COVID-19 patients. J Thromb Haemost JTH. https://doi.org/10.1111/jth.14876

16. Zhou F, Yu T, Du R et al (2020) Clinical course and risk factors for mortality of adult inpatients with COVID-19 in Wuhan, China: a retrospective cohort study. Lancet 395:1054-1062. https://doi. org/10.1016/S0140-6736(20)30566-3 
17. Tang N, Li D, Wang X, Sun Z (2020) Abnormal coagulation parameters are associated with poor prognosis in patients with novel coronavirus pneumonia. J Thromb Haemost JTH 18:844847. https://doi.org/10.1111/jth.14768

18. Zhang J-J, Dong X, Cao Y-Y et al (2020) Clinical characteristics of 140 patients infected with SARS-CoV-2 in Wuhan, China. Allergy. https://doi.org/10.1111/all.14238

19. Gao Y, Li T, Han M et al (2020) Diagnostic utility of clinical laboratory data determinations for patients with the severe COVID-19. J Med Virol. https://doi.org/10.1002/jmv.25770

20. Lippi G, Favaloro EJ (2020) D-dimer is Associated with Severity of Coronavirus Disease 2019: A Pooled Analysis. Thromb Haemost 120:876-878. https://doi.org/10.1055/s-0040-1709650

21. Ferreira FL, Bota DP, Bross A et al (2001) Serial evaluation of the SOFA score to predict outcome in critically ill patients. JAMA 286:1754-1758. https://doi.org/10.1001/jama.286.14.1754

22. de Grooth H-J, Geenen IL, Girbes AR et al (2017) SOFA and mortality endpoints in randomized controlled trials: a systematic review and meta-regression analysis. Crit Care Lond Engl 21:38. https://doi.org/10.1186/s13054-017-1609-1

23. Helms J, Tacquard C, Severac F et al (2020) High risk of thrombosis in patients with severe SARS-CoV-2 infection: a multicenter prospective cohort study. Intensive Care Med. https://doi. org/10.1007/s00134-020-06062-x

24. Huang C, Wang Y, Li X et al (2020) Clinical features of patients infected with 2019 novel coronavirus in Wuhan, China. Lancet Lond Engl 395:497-506. https://doi.org/10.1016/S0140 -6736(20)30183-5

25. Zhang L, Yan X, Fan Q et al (2020) D-dimer levels on admission to predict in-hospital mortality in patients with Covid-19. J Thromb Haemost JTH. https://doi.org/10.1111/jth.14859

26. Han H, Yang L, Liu R et al (2020) Prominent changes in blood coagulation of patients with SARS-CoV-2 infection. Clin Chem Lab Med. https://doi.org/10.1515/cclm-2020-0188
27. Wu C, Chen X, Cai Y et al (2020) Risk Factors Associated With Acute Respiratory Distress Syndrome and Death in Patients With Coronavirus Disease 2019 Pneumonia in Wuhan, China. JAMA Intern Med. https://doi.org/10.1001/jamainternmed.2020.0994

28. Yin S, Huang M, Li D, Tang N (2020) Difference of coagulation features between severe pneumonia induced by SARS-CoV2 and non-SARS-CoV2. J Thromb Thrombolysis. https://doi. org/10.1007/s11239-020-02105-8

29. Mortality Analyses. In: Johns Hopkins Coronavirus Resour. Cent. https://coronavirus.jhu.edu/data/mortality. Accessed 15 Jun 2020

30. Moher D, Schulz KF, Altman DG, CONSORT GROUP (Consolidated Standards of Reporting Trials) (2001) The CONSORT statement: revised recommendations for improving the quality of reports of parallel-group randomized trials. Ann Intern Med 134:657-662. https://doi.org/10.7326/0003-4819-134-8-20010 4170-00011

31. Moher D, Schulz KF, Altman D, CONSORT Group (Consolidated Standards of Reporting Trials) (2001) The CONSORT statement: revised recommendations for improving the quality of reports of parallel-group randomized trials. JAMA 285:1987-1991. https:// doi.org/10.1001/jama.285.15.1987

32. Moher D, Schulz KF, Altman DG (2001) The CONSORT statement: revised recommendations for improving the quality of reports of parallel-group randomised trials. Lancet Lond Engl 357:1191-1194

Publisher's Note Springer Nature remains neutral with regard to jurisdictional claims in published maps and institutional affiliations. 